\title{
Desmedipham (Pesticides)
}

\author{
Summary
}

\author{
Food Safety Commission of Japan
}

Food Safety Commission of Japan (FSCJ) conducted a risk assessment of desmedipham (CAS No. 13684-56-5), a carbanilate herbicides, based on results from various studies. Major adverse effects of desmedipham were suppressed body weight, hemolytic anemia, methemoglobinemia and follicular cell hypertrophy in thyroid. Neither carcinogenicity, reproductive toxicity, nor genotoxicity relevant to human health was observed on desmedipham. Desmedipham, at the dose with maternal toxicity, caused external anomalies such as mandibular malformation and cleft palate, visceral anomalies such as ventricular septum defect, and skeletal anomalies such as defect of sternum and asymmetric alignment of seternebral hemicentres in developmental toxicity studies in rats. No teratogenetic effects were observed in rabbits. The lowest no-observed-effect level (NOAEL) obtained in all studies was $3.2 \mathrm{mg} / \mathrm{kg}$ bw/day in a two-year combined chronic toxicity/carcinogenicity in rats. FSCJ specified an acceptable (ADI) of $0.032 \mathrm{mg} / \mathrm{kg}$ bw/day, applying a safety factor of 100 to the NOAEL. The lowest NOAEL for adverse effects elicited by a single oral administration of desmedipham was $90 \mathrm{mg} / \mathrm{kg}$ bw/day obtained from the developmental toxicity study in rabbits (the $2^{\text {nd }}$ study in the Table 2). Consequently, FSCJ specified an acute reference dose (ARfD) of $0.9 \mathrm{mg} / \mathrm{kg}$ bw applying a safety factor of 100 to the NOAEL.

\section{Conclusion in Brief}

Food Safety Commission of Japan (FSCJ) conducted a risk assessment of desmedipham (CAS No. 13684-56-5), a carbanilate herbicides, based on results from various studies.

The data used in the assessment include on the fate in animals (rats, cattle and chickens), fate in plants (sugar beets), residues in crops, subacute toxicity (rats and dogs), chronic toxicity (rats and dogs), combined chronic toxicity/carcinogenicity (rats), carcinogenicity (rats and mice), two-generation reproductive toxicity (rats), developmental toxicity (rats and rabbits), genotoxicity, and a mechanism of hemolytic anemia in dogs.

Major adverse effects of desmedipham were suppressed body weight, hemolytic anemia, methemoglobinemia and follicular cell hypertrophy in thyroid. Neither carcinogenicity, reproductive toxicity, nor genotoxicity relevant to human health was observed on desmedipham.
Desmedipham, at the dose with maternal toxicity, caused external anomalies such as mandibular malformation and cleft palate, visceral anomalies such as ventricular septum defect, and skeletal anomalies such as defect of sternum and asymmetric alignment of seternebral hemicentres in developmental toxicity studies in rats. No teratogenetic effects were observed in rabbits.

Based on the data on various studies, desmedipham (parent compound only) was identified as the substance relevant to the residue definition for dietary risk assessment in agricultural products.

The lowest no-observed-effect level (NOAEL) obtained in all studies was $3.2 \mathrm{mg} / \mathrm{kg}$ bw/day in a two-year combined chronic toxicity/carcinogenicity in rats. FSCJ specified an acceptable (ADI) of $0.032 \mathrm{mg} / \mathrm{kg}$ bw/day, applying a safety factor of 100 to the NOAEL.

The lowest NOAEL for adverse effects elicited by a single oral administration of desmedipham was $90 \mathrm{mg} / \mathrm{kg}$ bw/day

Published online: 28 September 2018

This is an English translation of excerpts from the original full report (July 2017-FS/452/2017). Only original Japanese texts have legal effect.

The original full report is available in Japanese at http://www.fsc.go.jp/fsciis/attachedFile/download?retrievalId=kya20171012112\&fileId=201 Acknowledgement: FSCJ wishes to thank the members of Expert Committee on Pesticides for the preparation of the original full report. Suggested citation: Food Safety Commission of JAPAN. Desmedipham: Summary. Food Safety. 2018; 6 (2): 130-135.

doi:10.14252/foodsafetyfscj.2017018s 
obtained from the developmental toxicity study in rabbits an acute reference dose (ARfD) of $0.9 \mathrm{mg} / \mathrm{kg}$ bw applying a (the $2^{\text {nd }}$ study in the Table 2). Consequently, FSCJ specified safety factor of 100 to the NOAEL. 
Table 1. Levels relevant to toxicological evaluation of desmedipham

\begin{tabular}{|c|c|c|c|}
\hline Species & Study & $\begin{array}{c}\text { Dose } \\
(\mathrm{mg} / \mathrm{kg} \mathrm{bw} / \text { day) }\end{array}$ & $\begin{array}{l}\text { NOAEL (mg/kg bw/day) } \\
\text { and critical endpoints }{ }^{\mathrm{a}}\end{array}$ \\
\hline \multirow{12}{*}{ Rat } & \multirow{2}{*}{$\begin{array}{l}\text { 13-week subacute } \\
\text { toxicity study } \\
\text { (the } 1^{\text {st }} \text { study) }\end{array}$} & $0,6,30,60,300 \mathrm{ppm}$ & $\begin{array}{l}\text { M: } 5.2 \\
\text { F: } 5.6 \\
\text { F/M: Increase in MetHb, and Ret, etc. }\end{array}$ \\
\hline & & $\begin{array}{l}\text { M: } 0,0.5,2.6,5.2,26 \\
F: 0,0.5,2.7,5.6,27\end{array}$ & \\
\hline & \multirow{2}{*}{$\begin{array}{l}\text { 13-week subacute } \\
\text { toxicity study } \\
\text { (the } 2^{\text {nd }} \text { study) }\end{array}$} & $0,160,800,4000 \mathrm{ppm}$ & $\begin{array}{l}\text { M: } 10.6 \\
\text { F: } 12.3 \\
\text { M: Congestive spleen, etc. } \\
\text { F: Decrease in } \mathrm{RBC}, \mathrm{Ht} \text { and } \mathrm{Hb} \text {, etc. }\end{array}$ \\
\hline & & $\begin{array}{l}\text { M: } 0,10.6,54,275 \\
\text { F: } 0,12.3,60,339\end{array}$ & \\
\hline & \multirow{2}{*}{$\begin{array}{l}\text { One-year chronic } \\
\text { toxicity study }\end{array}$} & $0,100,400,1200 \mathrm{ppm}$ & $\begin{array}{l}\text { M: } 6.5 \\
\text { F: } 31.7 \\
\text { F/M: Increase in T.Bil, etc. }\end{array}$ \\
\hline & & $\begin{array}{l}\text { M: } 0,6.5,25.2,75.0 \\
F: 0,7.9,31.7,97.1\end{array}$ & \\
\hline & \multirow[b]{2}{*}{$\begin{array}{l}\text { Two-year chronic } \\
\text { toxicity/ } \\
\text { carcinogenicity } \\
\text { study }\end{array}$} & $0,60,300,1500 \mathrm{ppm}$ & $\begin{array}{l}\text { M: } 3.2 \\
\text { F: } 3.9 \\
\text { F/M: Increase in MetHb and Ret, etc }\end{array}$ \\
\hline & & $\begin{array}{l}\text { Chronic toxicity study } \\
\text { M: } 0,3.2,15.7,79.9 \\
\text { F: } 0,3.9,19.8,101 \\
\text { Carcinogenicity study } \\
\text { M: } 0,3.3,16.1,84.0 \\
\text { F: } 0,4.1,20.2,104\end{array}$ & (Not carcinogenic) \\
\hline & \multirow[t]{2}{*}{$\begin{array}{l}\text { Two-year } \\
\text { carcinogenicity } \\
\text { study }\end{array}$} & $0,100,400,1200 \mathrm{ppm}$ & $\begin{array}{l}\text { M: } 5.4 \\
\text { F: } 6.8 \\
\text { M: Increase in alveolar macrophage } \\
\text { F: Increase in T.Bil } \\
\text { (Not carcinogenic) }\end{array}$ \\
\hline & & $\begin{array}{l}\text { M: } 0,5.4,21.6,64.4 \\
F: 0,6.8,28.4,86.6\end{array}$ & \\
\hline & \multirow[t]{2}{*}{$\begin{array}{l}\text { Two-generation } \\
\text { reproductive } \\
\text { toxicity study } \\
\text { (the } 1^{\text {st }} \text { study) }\end{array}$} & $0,50,250,1250 \mathrm{ppm}$ & $\begin{array}{l}\text { Parent and Offspring: } \\
\text { PM: } 4.0 \\
\text { PF: } 4.6 \\
\text { F1M: } 4.4 \\
\text { F1F: } 4.9 \\
\text { Parent: } \\
\text { F/M: Hemosiderin deposition in spleen } \\
\text { Offspring: Suppressed body weight } \\
\text { (No effect on reproduction) }\end{array}$ \\
\hline & & $\begin{array}{l}\text { PM: } 0,4.0,20.5,106 \\
\text { PF: } 0,4.6,23.3,120 \\
\text { F1M: } 0,4.4,22.5,118 \\
\text { F1F: } 0,4.9,25.3,130\end{array}$ & \\
\hline
\end{tabular}


Table 1. Levels relevant to toxicological evaluation of desmedipham (continued)

\begin{tabular}{|c|c|c|c|}
\hline Species & Study & $\begin{array}{c}\text { Dose } \\
\text { (mg/kg bw/day) }\end{array}$ & $\begin{array}{l}\text { NOAEL (mg/kg bw/day) } \\
\text { and critical endpoints }{ }^{\mathrm{a}}\end{array}$ \\
\hline \multirow{5}{*}{ Rat } & \multirow[t]{2}{*}{$\begin{array}{l}\text { Two-generation } \\
\text { reproductive } \\
\text { toxicity study } \\
\text { (the } 2^{\text {nd }} \text { study) }\end{array}$} & $0,100,400,1200 \mathrm{ppm}$ & $\begin{array}{l}\text { Parent and Offspring: } \\
\text { PM: } 32.5 \\
\text { PF: } 38.8 \\
\mathrm{~F}_{1} \mathrm{M}: 37.6 \\
\mathrm{~F}_{1} \mathrm{~F}: 42.5 \\
\text { Parent: } \\
\text { F/M: Suppressed body weight and decreased feed consumption } \\
\text { Offspring: lower weight (at birth) /Suppressed body weight } \\
\text { (during lactation period) } \\
\text { (No effect on reproduction) }\end{array}$ \\
\hline & & $\begin{array}{l}\text { PM: } 0,8.04,32.5,97.0 \\
\text { PF: } 0,9.67,38.8,118 \\
F_{1} M: 0,9.31,37.6,117 \\
F_{1} F: 0,10.5,42.5,128\end{array}$ & \\
\hline & $\begin{array}{l}\text { Developmental } \\
\text { toxicity study } \\
\text { (the } 1^{\text {st }} \text { study) }\end{array}$ & $0,10,100,1000$ & $\begin{array}{l}\text { Maternal: } 100 \\
\text { Embryo/fetus: } 100 \\
\text { Maternal: Suppressed body weight and decreased feed consumption } \\
\text { Embryo/fetus: External anomalies such as cleft palate and } \\
\text { mandibular malformation }\end{array}$ \\
\hline & $\begin{array}{l}\text { Developmental } \\
\text { toxicity study } \\
\text { (the } 2^{\text {nd }} \text { study) }\end{array}$ & $0,10,100,500$ & $\begin{array}{l}\text { Maternal: } 10 \\
\text { Embryo/fetus: } 100 \\
\text { Maternal: Increase in MetHb } \\
\text { Embryo/fetus: lower body weight, etc. }\end{array}$ \\
\hline & $\begin{array}{l}\text { Developmental } \\
\text { toxicity study } \\
\text { (the } 3^{\text {rd }} \text { study) }\end{array}$ & $0,60,250,1000$ & $\begin{array}{l}\text { Maternal: } 60 \\
\text { Embryo/fetus: } 250 \\
\text { Maternal: Increase in absolute spleen weight } \\
\text { Embryo/fetus: lower body weight and external anomalies such as cleft } \\
\text { palate }\end{array}$ \\
\hline \multirow{4}{*}{ Mouse } & & $0,30,150,750 \mathrm{ppm}$ & $\begin{array}{l}\text { M: } 21.7 \\
\text { F: } 30.8\end{array}$ \\
\hline & $\begin{array}{l}\text { Two-year } \\
\text { carcinogenicity } \\
\text { study }\end{array}$ & $\begin{array}{l}\text { Intermittent sacrifice } \\
\text { M: } 0,4.24,22.7,141 \\
\text { F: } 0,6.25,34.3,187 \\
\text { Carcinogenicity study } \\
\text { M: } 0,4.2,21.7,109 \\
\text { F: } 0,5.8,30.8,145\end{array}$ & $\begin{array}{l}\text { M: Increase in MetHb, etc. } \\
\text { F: Decrease in } \mathrm{Hb} \text { and } \mathrm{Ht} \text {, etc. } \\
\text { (Not carcinogenic) }\end{array}$ \\
\hline & \multirow{2}{*}{$\begin{array}{c}80 \text {-week } \\
\text { carcinogenicity } \\
\text { study }\end{array}$} & $0,400,1000,2500 \mathrm{ppm}$ & $\begin{array}{l}\text { M: } 60.8 \\
\text { F: } 71.9\end{array}$ \\
\hline & & $\begin{array}{l}\text { M: } 0,60.8,153,403 \\
\text { F: } 0,71.9,178,503\end{array}$ & $\begin{array}{l}\text { F/M: Necrosis of hepatocytes } \\
\text { (Not carcinogenic) }\end{array}$ \\
\hline
\end{tabular}


Table 1. Levels relevant to toxicological evaluation of desmedipham (continued)

\begin{tabular}{|c|c|c|c|}
\hline Species & Study & $\begin{array}{c}\text { Dose } \\
(\mathrm{mg} / \mathrm{kg} \text { bw/day })\end{array}$ & $\begin{array}{l}\text { NOAEL (mg/kg bw/day) } \\
\text { and critical endpoints }^{\mathrm{a}}\end{array}$ \\
\hline \multirow[b]{2}{*}{ Rabbit } & $\begin{array}{l}\text { Developmental } \\
\text { toxicity study } \\
\text { (the } 1^{\text {st }} \text { study) }\end{array}$ & $0,50,150,450$ & $\begin{array}{l}\text { Maternal: } 150 \\
\text { Embryo/fetus: } 50 \\
\text { Maternal: Suppressed body weight and feed consumption } \\
\text { Embryo/fetus: lower body weight } \\
\text { (Not teratogenic) }\end{array}$ \\
\hline & $\begin{array}{l}\text { Developmental } \\
\text { toxicity study } \\
\text { (the } 2^{\text {nd }} \text { study) }\end{array}$ & $0,30,90,270$ & $\begin{array}{l}\text { Maternal: } 30 \\
\text { Embryo/fetus: } 90 \\
\text { Maternal: Increase in absolute weight of spleen } \\
\text { Embryo/fetus: lower body weight, delayed ossification of sternum } \\
\text { and incompletely ossified phalanges } \\
\text { (Not teratogenic) }\end{array}$ \\
\hline \multirow{8}{*}{ Dog } & \multirow[b]{2}{*}{$\begin{array}{l}\text { 13-week subacute } \\
\text { toxicity study } \\
\text { (the } 1^{\text {st }} \text { study) }\end{array}$} & $0,1,5,150 \mathrm{ppm}$ & M: 4.97 \\
\hline & & $\begin{array}{l}\text { M: } 0,0.035,0.17,4.97 \\
F: 0,0.035,0.19,5.50\end{array}$ & $\begin{array}{l}\text { M: } 4.97 \\
\text { F: } 5.50 \\
\text { F/M: No toxicity }\end{array}$ \\
\hline & \multirow{2}{*}{$\begin{array}{l}\text { 13-week subacute } \\
\text { toxicity study } \\
\text { (the } 2^{\text {nd }} \text { study) }\end{array}$} & $0,100,500,1500 \mathrm{ppm}$ & $\begin{array}{l}\text { M: } 18.6 \\
\text { F: } 4.22\end{array}$ \\
\hline & & $\begin{array}{l}\text { M: } 0,3.73,18.6,55.6 \\
\text { F: } 0,4.22,21.0,62.2\end{array}$ & F/M: Follicular cell hypertrophy, etc. \\
\hline & \multirow{2}{*}{$\begin{array}{l}\text { One-year chronic } \\
\text { toxicity study }\end{array}$} & $\begin{array}{l}0,300,1500,7500 / 5000^{\mathrm{b}} \\
\mathrm{ppm}\end{array}$ & $\begin{array}{l}\text { M: } 9.7 \\
\text { F: } 10.4\end{array}$ \\
\hline & & $\begin{array}{l}\text { M: } 0,9.7,52.5,168 \\
\text { F: } 0,10.4,57.4,201\end{array}$ & F/M: Hemolytic anemia, etc. \\
\hline & \multirow[b]{2}{*}{$\begin{array}{l}\text { Effect on MetHb } \\
\text { formation }\end{array}$} & $\begin{array}{l}0,75,150,200,300,500, \\
1500 \mathrm{ppm}\end{array}$ & $\begin{array}{l}\text { M: } 15.5 \\
\text { F: } 11.1\end{array}$ \\
\hline & & $\begin{array}{l}\text { M: } 0,2.5,5.1,6.5,9.7,15.5 \\
45.0 \\
\text { F: } 0,2.5,4.3,5.3,11.1,15.7 \\
49.2\end{array}$ & \\
\hline \multicolumn{3}{|c|}{ ADI } & $\begin{array}{l}\text { NOAEL: } 3.2 \\
\text { SF: } 100 \\
\text { ADI: } 0.032\end{array}$ \\
\hline \multicolumn{3}{|c|}{ The critical study for setting ADI } & Two-year combined chronic toxicity/carcinogenicity study in rats \\
\hline
\end{tabular}

M, Male; F, Female; F/M, both sexes; PM, Male in Parent (P) generation; PF, Female in P generation; $F_{1} M$, Male in $F_{1}$ generation; $F_{1} F$, Female in $\mathrm{F}_{1}$ generation; ADI, Acceptable daily intake; $\mathrm{cRfD}$, Chronic reference dose; SF, Safety factor; UF, Uncertainty factor; NOAEL, No-observed-adverse-effect level; -, NOAEL could not be specified; MetHB, methohemoglobin; Ret, Reticulocytes; Ht, Hemotocrit; T.Bil, Total bilirubin

${ }^{\text {a }}$, The adverse effect observed at the lowest-observed-adverse-effect level (LOAEL); ${ }^{\text {, }}$ Administered at 7500 ppm for the first 28 days, (reduced to) $5000 \mathrm{ppm}$ after 28 days 
Table 2. Potential adverse effects of a single oral administration of desmedipham

\begin{tabular}{|c|c|c|c|}
\hline Species & Study & $\begin{array}{c}\text { Dose } \\
(\mathrm{mg} / \mathrm{kg} \mathrm{bw} / \text { day })\end{array}$ & $\begin{array}{l}\text { NOAEL (mg/kg bw/day) } \\
\text { and critical endpoints }{ }^{\mathrm{a}}\end{array}$ \\
\hline \multirow{4}{*}{ Rat } & Acute toxicity study & 5000 & $\begin{array}{l}\text { F/M: - } \\
\text { F/M: Sedation, dyspnea, flexion, suppressed body weight, } \\
\text { rough fur and blanching }\end{array}$ \\
\hline & $\begin{array}{l}\text { Developmental toxicity study } \\
\text { (the } 1^{\text {st }} \text { study) }\end{array}$ & $0,10,100,1000$ & $\begin{array}{l}\text { Maternal: } 100 \\
\text { Maternal: Suppressed body weight and decreased feed } \\
\text { consumption }\end{array}$ \\
\hline & $\begin{array}{l}\text { Developmental toxicity study } \\
\text { (the } 2^{\text {nd }} \text { study) }\end{array}$ & $0,10,100,500$ & $\begin{array}{l}\text { Maternal: } 100 \\
\text { Maternal: Suppressed body weight and decreased feed } \\
\text { consumption }\end{array}$ \\
\hline & $\begin{array}{l}\text { Developmental toxicity study } \\
\text { (the } 3^{\text {rd }} \text { study) }\end{array}$ & $0,60,250,1000$ & $\begin{array}{l}\text { Maternal: } 250 \\
\text { Maternal: Suppressed body weight and decreased feed } \\
\text { consumption }\end{array}$ \\
\hline Mouse & Acute toxicity study & 3500 & $\begin{array}{l}\text { M: } 3500 \\
\text { F: - } \\
\text { F: Dyspnea, hypothermia and coma }\end{array}$ \\
\hline \multirow{2}{*}{ Rabbit } & $\begin{array}{l}\text { Developmental toxicity study } \\
\text { (the } 1^{\text {st }} \text { study) }\end{array}$ & $0,50,150,450$ & $\begin{array}{l}\text { Maternal: } 150 \\
\text { Maternal: Suppressed body weight and feed consumption }\end{array}$ \\
\hline & $\begin{array}{l}\text { Developmental toxicity study } \\
\text { (the } 2^{\text {nd }} \text { study) }\end{array}$ & $0,30,90,270$ & $\begin{array}{l}\text { Maternal: } 90 \\
\text { Maternal: Suppressed body weight and feed consumption }\end{array}$ \\
\hline \multicolumn{3}{|c|}{ ARfD } & $\begin{array}{l}\text { NOAEL: } 90 \\
\text { SF: } 100 \\
\text { ARfD: } 0.9\end{array}$ \\
\hline \multicolumn{3}{|c|}{ The critical study for setting ARfD } & Developmental toxicity study in rabbits (the $2^{\text {nd }}$ study) \\
\hline
\end{tabular}

ARfD, Acute reference dose; SF, Safety factor; NOAEL, No-observed-adverse-effect level; -, NOAEL could not be specified

a , The adverse effect observed at LOAEL 